\title{
Pacific
}

Journal of

Mathematics

\section{GEOMETRIC ASPECTS OF BÄCKLUND TRANSFORMATIONS} OF WEINGARTEN SUBMANIFOLDS

STEVEn Buyske 


\title{
GEOMETRIC ASPECTS OF BÄCKLUND TRANSFORMATIONS OF WEINGARTEN SUBMANIFOLDS
}

\author{
Steven G. Buyske
}

\begin{abstract}
If $f_{1}$ and $f_{2}$ are immersions of an $n$-manifold $M$ into $\mathbf{R}^{2 n-1}$ such that their induced frame bundles differ by a constant right action, then $f_{1}$ and $f_{2}$ both satisfy a Weingarten condition on their normal bundles and the right action corresponds to a generalization of the classical Bäcklund transformation.
\end{abstract}

1. Introduction. In the 1890 s Bianchi, Lie, and finally Bäcklund looked at what are now called Bäcklund transformations of surfaces. In modern parlance, they begin with two surfaces in Euclidean space in a line congruence: there is a mapping between the surfaces $M_{1}$ and $M_{2}$ such that the line through any two corresponding points is tangent to both surfaces. Bäcklund proved that if a line congruence satisfied two additional conditions, that the line segment joining corresponding points has constant length, and that the normals at corresponding points form a constant angle, then the two surfaces are necessarily surfaces of constant negative curvature. He was also able to show that a Bäcklund transformation is integrable, in the sense that given a point on a surface of constant negative curvature and a tangent line segment at that point, a new surface of constant negative curvature can be found, containing the endpoint of the line segment, that is a Bäcklund transform of the original surface.

Since that time, much of the attention has focused on Bäcklund transformations as transformations of solutions to partial differential equations. Since a surface of constant negative curvature is equivalent to a solution of the Sine-Gordon equation, the Bäcklund 
transformation of surfaces corresponds to a Bäcklund transformation of the solution to the Sine-Gordon equation. In 1980, Tenenblat and Terng [5] returned to the geometric viewpoint and showed that the Bäcklund transformation could be extended to $n$-dimensional submanifolds of $2 n-1$-dimensional Euclidean space. Under the conditions that the connecting tangent line segment have constant length, that the normal affine spaces meet at an isoclinic, constant angle, and that the normal bundle be flat, they showed that the submanifolds have to have constant negative curvature. They also showed that the Bäcklund transformation is integrable.

Darboux (Chapter 12 of Book 7 in [2]) and Bryant [1] generalized the Bäcklund transformation in a different way. They relaxed the condition that the connecting line segment between the surfaces be tangent to both surfaces, and instead required that the segment make a constant angle with each tangent plane. In this case, the surfaces need no longer have constant negative curvature; instead, they must satisfy a linear Weingarten relation of the form $\alpha K \pm$ $2 \beta H+\gamma=0$. In this paper we extend Darboux's and Bryant's work to $n$-dimensional submanifolds of $2 n-1$-Euclidean space. The author would like to thank the referee for many helpful comments about the exposition of this paper.

2. Preliminaries. Let $\mathbf{E}(2 n-1)$ be the group of rigid Euclidean motions of $\mathbf{R}^{2 n-1}$. We will consider $\mathbf{E}(2 n-1)$ to be the oriented orthonormal frame bundle $\mathbf{E}(2 n-1) \rightarrow \mathbf{R}^{2 n-1}$, and represent it as the group of matrices of the form

$$
\left(\begin{array}{ll}
D & p \\
0 & 1
\end{array}\right) \text { where } D \in O(2 n-1) \text { and } p \in \mathbf{R}^{2 n-1}
$$

The left-invariant Maurer-Cartan one-forms $\left\{\theta^{I}, \theta_{J}^{I}\right\}$ on $\mathbf{E}(2 n-1)$ satisfy the structure equations

$$
\begin{aligned}
& d \theta^{I}=-\theta_{J}^{I} \wedge \theta^{J} \\
& d \theta_{J}^{I}=-\theta_{K}^{I} \wedge \theta_{J}^{K}
\end{aligned}
$$

where here, as in the sequel, repeated indices are summed over and 
$1 \leq I, J, K \leq 2 n-1$. We can write

$$
\theta=\left(\begin{array}{cc}
\theta_{J}^{I} & \theta^{I} \\
0 & 0
\end{array}\right)
$$

for convenience.

Suppose $f: M \rightarrow \mathbf{R}^{2 n-1}$ is an immersion with a locally flat normal bundle. We can pull back the frame bundle $\mathbf{E}(2 n-1)$ to obtain a principal bundle over $M$. If $\mathcal{F}: M \rightarrow f^{*} \mathbf{E}(2 n-1)$ is a section of this bundle, then the forms $\omega^{I}=\mathcal{F}^{*} \theta^{I}$ and $\omega_{J}^{I}=\mathcal{F}^{*} \theta_{J}^{I}$ satisfy

$$
\begin{aligned}
d f & =e_{I} \omega^{I} \\
d e_{I} & =e_{J} \omega_{I}^{J} .
\end{aligned}
$$

We restrict the bundle $f^{*} \mathbf{E}(2 n-1)$ so that all sections have the following two properties:

(a) The frames must be adapted; that is,

$$
f_{*} T_{p} M=\operatorname{span}\left\{e_{1}, \ldots, e_{n}\right\} .
$$

Equivalently, $\omega^{\alpha}=0$ on each section, for $\alpha>n$.

(b) The normal components of the frames are locally parallel. Equivalently, $\omega_{\beta}^{\alpha}=0$ on each section, for $\alpha, \beta>n$. This is possible because the immersion $f$ has a flat normal bundle.

We will call a section of $f^{*} \mathbf{E}(2 n-1)$ that satisfies condition (1) a framed map, while a section of $f^{*} \mathrm{E}(2 n-1)$ satisfying (1) and (2) will be called a framed map with flat normal bundle.

We need to define angles between two $m$ planes in a $2 m$-dimensional inner product space $(V,())$. Following [5], let $E$ and $E^{\prime}$ be $m$ planes in $V$, and let $P: V \rightarrow E$ be the orthogonal projection. We can define a symmetric bilinear form on $E^{\prime}$ by $\left\langle v_{1}, v_{2}\right\rangle=$ $\left(P\left(v_{1}\right), P\left(v_{2}\right)\right)$. There is a self-adjoint operator $T: E^{\prime} \rightarrow E^{\prime}$ such that $\left\langle v_{1}, v_{2}\right\rangle=\left(T v_{1}, v_{2}\right)$. The $m$ angles between $E$ and $E^{\prime}$ are the angles $\phi_{1}, \ldots, \phi_{m}$ such that $\cos ^{2} \phi_{1}, \ldots, \cos ^{2} \phi_{m}$ are the eigenvalues for $T$. Geometrically, there is a unit vector $v_{m}$ such that the angle, $\phi_{m}$, between $v_{m}$ and $P\left(v_{m}\right)$ is the largest among the angles between unit vectors in $E^{\prime}$ and their projections to $E$. The angle $\phi_{m-1}$ is the largest angle between a unit vector in $E^{\prime} \cap v_{m}^{\perp}$ and its projection, and so on. When all of these angles are equal, the planes are called 
isoclinic. In any case, there are two orthogonal bases $e_{1}, \ldots, e_{2 m}$ and $\tilde{e}_{1}, \ldots, \tilde{e}_{2 m}$ of $V$ such that $\tilde{e}_{1}, \ldots, \tilde{e}_{m}$ are eigenvectors of $T$ with eigenvalues $\cos ^{2} \phi_{1}, \ldots, \cos ^{2} \phi_{m}$, the vectors $e_{1}, \ldots e_{m}$ form a basis for $E$, and

$$
\begin{aligned}
\tilde{e}_{i} & =\cos \phi_{i} e_{i}+\sin \phi_{i} e_{m+i-1} \\
\tilde{e}_{m+i-1} & =-\sin \phi_{i} e_{i}+\cos \phi_{i} e_{m+i-1}
\end{aligned}
$$

\section{Bäcklund Transformations and Weingarten Submani-} folds. Consider two immersions $f_{1}, f_{2}: M^{n} \rightarrow \mathbf{R}^{2 n-1}$ with flat normal bundles, and let $\mathcal{F}_{1}$ and $\mathcal{F}_{2}$ be sections satisfying conditions (1) and (2) into their respective bundles. Suppose $\mathcal{F}_{1}$ and $\mathcal{F}_{2}$ differ by a fixed right action $A \in \mathbf{E}(2 n-1)$, so that

$$
\mathcal{F}_{2}=R_{A} \circ \mathcal{F}_{1}
$$

If the normal spaces to the immersions at corresponding points span a $2 n$-2-dimensional subspace of $\mathbf{R}^{2 n-1}$, then by an appropriate left action on both $\mathcal{F}_{1}$ and $\mathcal{F}_{2}$ by $O(2 n-1) \subset \mathbf{E}(2 n-1)$, we may assume without loss of generality that $A$ is of the form

$$
A=\left(\begin{array}{cccc}
-1 & 0 & 0 & r^{1} \\
0 & C & S & r^{i} \\
0 & S & -C & r^{n-1+i} \\
0 & 0 & 0 & 1
\end{array}\right)
$$

where $2 \leq i \leq n$,

$$
\begin{aligned}
C & =\left(\begin{array}{ccc}
\cos \phi_{2} & 0 & 0 \\
0 & \ddots & 0 \\
0 & 0 & \cos \phi_{n}
\end{array}\right), \\
S & =\left(\begin{array}{ccc}
\sin \phi_{2} & 0 & 0 \\
0 & \ddots & 0 \\
0 & 0 & \sin \phi_{n}
\end{array}\right),
\end{aligned}
$$

and $\sin \phi_{i} \neq 0$ for all $i$. The angles $\phi_{i}$ are the $n-1$ angles that describe how the normal planes to the two immersion meet. We can 
assume that the angles are listed in increasing order. The condition that $\sin \phi_{i} \neq 0$ is equivalent to the condition that the two normal spaces at corresponding points span a $2 n-2$-dimensional subspace. This may be thought of as requiring that the two normal spaces at corresponding points contain no parallel subspaces.

The equations

$$
\begin{aligned}
& \omega=\mathcal{F}_{1}^{*} \theta \\
& \tilde{\omega}=\mathcal{F}_{2}^{*} \theta=\mathcal{F}_{1}^{*} \circ R_{A}^{*} \theta
\end{aligned}
$$

imply

$$
\tilde{\omega}=A^{-1} \omega A .
$$

This equation yields the following relations, where $s_{i}$ and $c_{i}$ is used for $\sin \phi_{i}$ and $\cos \phi_{i}$, respectively:

$$
\omega_{j}^{1}=-s_{j} \omega_{n-1+j}^{1}-c_{j} \omega_{j}^{1}
$$

$$
\tilde{\omega}_{n-1+j}^{i}=-c_{i} s_{j} \omega_{n-1+j}^{n-1+i}+s_{i} s_{j} \omega_{j}^{n-1+i}-c_{i} c_{j} \omega_{n-1+j}^{i}+c_{i} s_{j} \omega_{j}^{i}
$$

$$
\begin{aligned}
\tilde{\omega}_{n-1+j}^{1} & =c_{j} \omega_{n-1+j}^{1}-s_{j} \omega_{j}^{1} \\
\tilde{\omega}_{j}^{i} & =s_{i} s_{j} \omega_{n-1+j}^{n-1+i}+s_{i} c_{j} \omega_{j}^{n-1+i}+c_{i} s_{j} \omega_{n-1+j}^{i}+c_{i} c_{j} \omega_{j}^{i}
\end{aligned}
$$

$$
\begin{aligned}
\tilde{\omega}_{n-1+j}^{n-1+i} & =c_{i} c_{j} \omega_{n-1+j}^{n-1+i}-c_{i} s_{j} \omega_{j}^{n-1+i}-s_{i} c_{j} \omega_{n-1+j}^{i}+s_{i} s_{j} \omega_{j}^{i} \\
\tilde{\omega}^{1} & =-\omega^{1}-r^{n-1+k} \omega_{n-1+k}^{1}-r^{k} \omega_{k}^{1} \\
\tilde{\omega}^{i} & =s_{i} \omega^{n-1+i}+r^{n-1+k} s_{i} \omega_{n-1+k}^{n-1+i}+r^{k} s_{i} \omega_{k}^{n-1+i}
\end{aligned}
$$$$
+r^{1} s_{i} \omega_{1}^{n-1+i}
$$

$$
+c_{i} \omega^{i}+c_{i} r^{n-1+k} \omega_{n-1+k}^{i}+c_{i} r^{k} \omega_{k}^{i}+c_{i} r^{1} \omega_{1}^{i}
$$

$$
\begin{aligned}
\tilde{\omega}^{n-1+i}=- & c_{i} \omega^{n-1+i}-c_{i} r^{n-1+k} \omega_{n-1+k}^{n-1+i}-c_{i} r^{k} \omega_{k}^{n-1+i} \\
& -c_{i} r^{1} \omega_{1}^{n-1+i}+s_{i} \omega^{i} \\
& +r^{n-1+k} s_{i} \omega_{n-1+k}^{i}+r^{k} s_{i} \omega_{k}^{i}+r^{1} s_{i} \omega_{1}^{i}
\end{aligned}
$$

where $2 \leq i, j, k \leq n$, and summation is over $k$, but not over $i$ or $j$. Because $\mathcal{F}_{1}$ and $\mathcal{F}_{2}$ satisfy conditions (1) and (2), we have

$$
\tilde{\omega}^{n-1+i}=\omega^{n-1+i}=\tilde{\omega}_{n-1+j}^{n-1+i}=\omega_{n-1+j}^{n-1+i}=0 .
$$


Taking the exterior derivative of equation (4.e), using equations (4.e) and (4.h) to eliminate $\omega_{j}^{i}$ and $\omega_{j}^{1}$, and using equation (5), we find

$$
\begin{aligned}
& 0=\frac{\left(r^{1}\right)^{2}}{s^{2} s^{j}} d \tilde{\omega}_{n-1+j}^{n-1+i} \\
&=-\left(r^{n-1+k}+\cot \phi_{k} r^{k}\right) \omega^{i} \wedge \omega_{j}^{n-1+k} \\
&-\left(r^{n-1+k}+\cot \phi_{k} r^{k}\right) \omega_{i}^{n-1+k} \wedge \omega^{j} \\
&+\left(r^{n-1+k}+\cot \phi_{k} r^{k}\right) \omega_{i}^{n-1+k} \wedge\left(r^{n-1+l}+\cot \phi_{l} r^{l}\right) \omega_{j}^{n-1+l} \\
&+\omega^{i} \wedge \omega^{j}+\frac{1}{\left(s^{k}\right)^{2}} \omega_{i}^{n-1+k} \wedge \omega_{j}^{n-1+k} .
\end{aligned}
$$

Similarly, we have

$$
\begin{aligned}
0= & \frac{r^{1}}{s^{i}} d \tilde{\omega}^{n-1+i}=-\left(r^{n-1+k}+\cot \phi_{k} r^{k}\right) \omega^{i} \wedge \omega_{1}^{n-1+k}= \\
& -\left(r^{n-1+k}+\cot \phi_{k} r^{k}\right) \omega_{i}^{n-1+k} \wedge=\omega^{1} \\
& +\left(r^{n-1+k}+\cot \phi_{k} r^{k}\right) \omega_{i}^{n-1+k} \wedge\left(r^{n-1+l}+\cot \phi_{l} r^{l}\right) \omega_{1}^{n-1+l} \\
& +\omega^{i} \wedge \omega^{1}+\frac{1}{\left(s^{k}\right)^{2}} \omega_{i}^{n-1+k} \wedge \omega_{1}^{n-1+k} .
\end{aligned}
$$

These equations can be simplified by using the parallel normal field

$$
\eta_{\bullet}=\sum_{k} \frac{r^{n-1+k}+\cot \phi_{k} r^{k}}{L} e_{n-1+k}
$$

where $L=\sqrt{\sum_{k}\left(r^{n-1+k}+\cot \phi_{k} r^{k}\right)^{2}}$. If we define $\omega_{a}^{\bullet}$ by the equation $d \eta_{\bullet}=-e_{a} \omega_{a}^{\bullet}$, then have

$$
\begin{aligned}
0= & \frac{\left(r^{1}\right)^{2}}{s^{2} s^{\jmath}} d \tilde{\omega}_{n-1+j}^{n-1+i} \\
= & -L \omega^{i} \wedge \omega_{j}^{\bullet}-L \omega_{i}^{\bullet} \wedge \omega^{j}+L^{2} \omega_{i}^{\bullet} \wedge \omega_{j}^{\bullet} \\
& \quad+\omega^{i} \wedge \omega^{\jmath}+\frac{1}{\left(s^{k}\right)^{2}} \omega_{\imath}^{n-1+k} \wedge \omega_{j}^{n-1+k}
\end{aligned}
$$


and

$$
\begin{aligned}
0= & \frac{r^{1}}{s^{i}} d \tilde{\omega}^{n-1+i} \\
= & -L \omega^{i} \wedge \omega_{1}^{\bullet}-L \omega_{i}^{\bullet} \wedge \omega^{1}+L^{2} \omega_{i}^{\bullet} \wedge \omega_{1}^{\bullet}+\omega^{i} \wedge \omega^{1} \\
& \quad+\frac{1}{\left(s^{k}\right)^{2}} \omega_{i}^{n-1+k} \wedge \omega_{1}^{n-1+k}
\end{aligned}
$$

If we write $I I^{\bullet}$ for the $\eta_{\bullet}$ component of the second fundamental form of $f_{1}$, then $I I^{\bullet}=\omega_{a}^{\bullet} \otimes \omega^{a}$. For any two-plane $\Pi \in T_{p} M$ with basis vectors $X$ and $Y$, define $\mathcal{K}_{\bullet}(\Pi)$ to be $I I^{\bullet}(X, X) I I^{\bullet}(Y, Y)-$ $I I^{\bullet}(X, Y)^{2}$ and $\mathcal{H}_{\bullet}(\Pi)$ to be $1 / 2\left(I I^{\bullet}(X, X)+I I^{\bullet}(Y, Y)\right)$. In other words, $\mathcal{K}_{\bullet}(\Pi)$ is the determinant of $I I^{\bullet}$ restricted to $\Pi$ and $\mathcal{H}_{\bullet}(\Pi)$ is one half the trace of $I I^{\bullet}$ to $\Pi$. If $\Pi$ has basis vectors $e_{a}$ and $e_{b}$, then we have $\omega_{a}^{\bullet} \wedge \omega_{b}^{\bullet}\left(e_{a}, e_{b}\right)=\mathcal{K}_{\bullet}(\Pi)$ and $\left(\omega_{a}^{\bullet} \wedge \omega^{b}+\omega^{a} \wedge \omega_{b}^{\bullet}\right)\left(e_{a}, e_{b}\right)=$ $2 \mathcal{H}_{.}(\Pi)$. Equations (6) and (7) then show that for any tangent two-plane $\Pi$ of $f(M)$,

$$
L^{2} \mathcal{K}_{\bullet}(\Pi) \pm 2 L \mathcal{H}_{\bullet}(\Pi)+1+\alpha^{*}(\Pi)=0
$$

where $\alpha^{*}$ is the 2 -form defined by

$$
\alpha^{*}=\sum_{k ; a<b} 1 /\left(s^{k}\right)^{2} \omega_{a}^{n-1+k} \wedge \omega_{b}^{n-1+k}
$$

Note that in the isoclinic case, where all the $s_{k}$ are equal, by the Gauss equation $\alpha^{*}$ is simply a scalar multiple of the submanifold's Riemannian curvature 2 -form (that is, the intrinsic curvature of the submanifold).

An obvious question here is whether $\eta_{\bullet}$ and $\alpha^{*}$ are genuinely geometric, and not dependent on the particular choice of frame. If the $s^{k}$ are distinct, then equation (1) uniquely determines the frame, and so $\eta_{\bullet}$ and $\alpha^{*}$. Now suppose $s^{i_{1}}=\cdots=s^{i_{m}}$. The space spanned by $e_{i_{1}}, \ldots, e_{i_{m}}$ is well-defined, as is the space spanned by $e_{n-1+i_{1}}, \ldots, e_{n-1+i_{m}}$. The projection of the vector $\overline{f_{2}(x) f_{1}(x)}$ onto $f_{2 *} T_{x} M$ and then back onto $\left(f_{1 *} T_{x} M\right)^{\perp}$ is $\sum_{k}\left(\left(s^{k}\right)^{2} r^{n-1+k}+\right.$ $\left.s^{k} c^{k} r^{k}\right) e_{n-1+k}$. The projection of this vector to the subspace spanned by $e_{n-1+i_{1}}, \ldots, e_{n-1+i_{m}}$ is well-defined, as is $1 /\left(s^{k}\right)^{2}$ times this vector. The normal field $\eta_{\bullet}$ simply lies in the direction of the sum (over $i)$ of these vectors. Note that in the case where all the $s_{k}$ are equal, 
$\eta$. is the unit normal in the direction of the projection of $\overline{f_{2}(x) f_{1}(x)}$ onto $f_{2 *} T_{x} M$ and then back onto $\left(f_{1 *} T_{x}^{M}\right)^{\perp}$. Since all the other terms in equation (8) are independent of the particular choice of frame, $\alpha^{*}(\Pi)$ is as well.

The situation for $\mathcal{F}_{2}$ can be calculated from the equation $\mathcal{F}_{1}=$ $R_{A^{-1}} \circ \mathcal{F}_{2}$. In this case,

$$
\eta_{\bullet}=\sum_{k} \frac{r^{k}}{s^{k} L} \tilde{e}_{n-1+k}
$$

where $L=\sqrt{\sum_{k}\left(r^{k} / s^{k}\right)^{2}}$.

We record the preceding discussion in the following theorem.

THEOREM 1. Let $\mathcal{F}_{1}, \mathcal{F}_{2}: M \rightarrow \mathbf{E}(2 n-1)$ be framed maps with flat normal bundles that differ by a fixed right action $A \in$ $\mathrm{E}(2 n-1)$. If none of the angles $\phi_{2}, \ldots, \phi_{n}$ between the normal spaces are zero, then $\mathcal{F}_{1}$ and $\mathcal{F}_{2}$ both satisfy a linear Weingarten condition. In particular, there exists a parallel normal field $\eta_{\bullet}$ and a constant $L$ such that for any tangent two-plane $\Pi$,

$$
L^{2} \mathcal{K}_{\bullet}(\Pi) \pm 2 L \mathcal{H}_{\bullet}(\Pi)+1+\alpha^{*}(\Pi)=0
$$

where $\mathcal{K}_{\bullet}(\Pi)$ and $\mathcal{H}_{\bullet}(\Pi)$ are the determinant and one half the trace of the $\eta_{\bullet}$ component of the second fundamental form of $f_{1}$ restricted to $\Pi$, and $\alpha^{*}$ is the 2-form defined in equation (9). If $\phi_{2}=\cdots=\phi_{n}$, then $\alpha^{*}$ is the Riemannian curvature 2-form of $M$ multiplied by $\csc ^{2} \phi_{2}$.

There is also an integrability theorem.

THEOREM 2. Let $M$ be a connected and simply connected smooth manifold and let $\mathcal{F}_{1}$ be a framed map of $M$ with flat normal bundle. Suppose there exist non-zero $(\bmod \pi)$ constants $\phi_{2}, \ldots, \phi_{n}$ such that there exists a parallel normal field $\eta_{\bullet}$ and an $L$ so that for any tangent two-plane $\Pi$ of $f_{1}(M)$,

$$
L^{2} \mathcal{K}_{\bullet}(\Pi) \pm 2 L \mathcal{H}_{\bullet}(\Pi)+1+\alpha^{*}(\Pi)=0
$$


where $\mathcal{K}_{\bullet}, \mathcal{H}_{\bullet}, \alpha^{*}$ are defined as in Theorem 3.. Then for any $A$ of the form

$$
A=\left(\begin{array}{cccc}
-1 & 0 & 0 & r^{1} \\
0 & C & S & r^{i} \\
0 & S & -C & r^{n-1+i} \\
0 & 0 & 0 & 1
\end{array}\right),
$$

with $C$ and $S$ defined in (2) and (3), such that $=\eta_{\bullet}=\sum_{k}\left(r^{n-1+k}+\right.$ $\left.\cot \phi_{k} r^{k}\right) / L e_{n-1+k}$, there exists a framed map with flat normal bundle $\mathcal{F}_{2}$ that differs from $\mathcal{F}_{1}$ by a right action by $A$.

Proof. Let $\mathcal{B} \rightarrow M$ be the bundle of adapted frames of the immersion $f: M \rightarrow \mathbf{R}^{2 n-1}$. The bundle $\mathcal{B}$ is an $O(n) \times O(n-1)$ principal bundle. We will look for adapted frame immersion as sections of this bundle. Define $\mu_{A}: \mathcal{B} \rightarrow \mathbf{E}(2 n-1)$ to be the natural inclusion of $\mathcal{B}$ in $\mathbf{E}(2 n-1)$ followed by right action by $A$. The forms $\tilde{\omega}^{I}$ and $\tilde{\omega}_{J}^{I}$ are related to the forms $\omega^{I}$ and $\omega_{J}^{I}$ by the equations in (4). The forms $\left\{\omega_{b}^{a}\right\}_{1 \leq a<b \leq n}$ and $\left\{\omega_{n-1+j}^{n-1+i}\right\}_{2 \leq i<j \leq n}$, being connection forms, are transverse to the fibers of $\mathcal{B} \rightarrow M$. Consequently, the forms $\left\{\tilde{\omega}_{n-1+j}^{n-1+i}\right\}_{2 \leq i<j \leq n}$ and $\left\{\tilde{\omega}^{n-1+i}\right\}_{2 \leq i \leq n}$ are also transverse. It is easy to check that they are independent. Let $\mathcal{I}$ be the ideal generated by $\left\{\tilde{\omega}_{n-1+j}^{n-1+i}, \tilde{\omega}^{n-1+i}, \omega_{n-1+j}^{n-1+i}\right\}$. Our assumptions and the calculations earlier guarantee that $d \mathcal{I} \subset \mathcal{I}$, so the distribution defined by $\mathcal{I}=0$ is integrable. Since the forms in $\mathcal{I}$ are transverse to the fiber, the foliation induced by $\mathcal{I}=0$ has leaves that project under $\mathcal{B} \rightarrow M$ as open mappings, which are therefore local diffeomorphisms. Since the fibers of $\mathcal{B} \rightarrow M$ are compact, and since $M$ is connected and simply connected, these leaves are in fact diffeomorphic to $M$. Therefore, for any section $\sigma: M \rightarrow \mathcal{B}$ such that $\sigma^{*} \mathcal{I}=0$, the map $\mu_{A} \circ \sigma: M \rightarrow \mathbf{R}^{2 n-1}$ is an adapted frame immersion.

REMARK 1 . In the case $n=2$ the two theorems specialize to results due to Bryant [1] . In the isoclinic case, where $s^{2}=\cdots=s^{n}$, if $r^{2}=\cdots=r^{n}=0$, the theorems specialize to results due to Tenenblat and Terng [5]. Theorem 2 is noticeably simpler in the isoclinic case, where $\alpha^{*}$ is simply the Riemann curvature tensor times the reciprocal of the sine of the isoclinic angle. 
REMARK 2. It is possible to develop the theory in this paper beginning not with an immersion $f$ into $\mathbf{R}^{2 n-1}$, but with an immersion $\mathcal{F}$ into $\mathbf{E}(2 n-1)$. In this case, the projection $\pi \circ \mathcal{F}$ of the manifold into $\mathbf{R}^{2 n-1}$ may not itself be an immersion, but it will be smoothly framed. Such a situation is studied by Gollek in [3] and [4] for the higher-dimensional Bäcklund transformation, and by Bryant [1] in greater generality. It is not difficult to extend the results of this paper to include such a setting.

4. An Example. There is a large class of isoclinic examples that are easy to generate. Begin with a constant negative curvature immersion of an $n$-manifold $M$. Set $r^{n-1+i}=0$ for all $i$, and set $\phi_{2}=\cdots=\phi_{n}=\pi / 2$. The normal field $\eta_{\bullet}$ is then the zero field. The framing of the immersion required for the Bäcklund transformation discussed here is exactly the same as the framing for the Bäcklund transformation for constant negative curvature submanifolds discussed in [5] and [6]. The vector between corresponding points of the immersion and its Bäcklund transform is tangent to the original immersion, but has both normal and tangential components to the transform.

For $n=3$, consider the mapping corresponding to the trivial solution of the generalized Sine-Gordon equation; this mapping is contained in a line. The Bäcklund transformation of this mapping with $r^{n-1+i}=0$ and $\phi_{i}=\pi / 2$ gives the following immersion:

$$
\begin{aligned}
1 / \cosh x & \left(\cos y\left(\cos m+r^{2} \cos m \sinh x-r^{3} \sin m \cosh x\right)\right. \\
& \sin y\left(\cos m+r^{2} \cos m \sinh x-r^{3} \sin m \cosh x\right) \\
& \cos z\left(\sin m+r^{2} \sin m \sinh x+r^{3} \cos m \cosh x\right) \\
& \sin z\left(\sin m+r^{2} \sin m \sinh x+r^{3} \cos m \cosh x\right) \\
& \left.-r^{2}+\sinh x-x \cosh x\right)
\end{aligned}
$$


The vector components of the framing are

$$
\left(\begin{array}{ccccc}
-\frac{\cos m \cos y}{\cosh x}-\sin y & 0 & \frac{\cos m \cos y \sinh x}{\cosh x}-\sin m \cos y \\
-\frac{\cos m \sin y}{\cosh x} & \cos y & 0 & \frac{\cos m \sin y \sinh x}{\cosh x}-\sin m \sin y \\
-\frac{\sin m \cos z}{\cosh x} & 0 & -\sin z & \frac{\sin m \cos z \sinh x}{\cosh x} & \cos m \cos z \\
-\frac{\sin m \sin z}{\cosh x} & 0 & \cos z & \frac{\sin m \sin z \sinh x}{\cosh x} & \cos m \sin z \\
-\frac{\sinh x}{\cosh x} & 0 & 0 & \frac{-1}{\cosh x} & 0
\end{array}\right) .
$$

\section{REFERENCES}

[1] R. L. Bryant, Generalized immersions and Bäcklund theory I, preprint, 1980.

[2] G. Darboux, Leçons sur la Théorie Générale des Surfaces, vol. III, Gauthier-Villars, Paris, 1896.

[3] H. Gollek, The 1-soliton solutions of the generalized sine-Gordon equation in dimension $n=3$, Proc. of the Conference on Differential Geom. and its Appl., Part 1 (Nove Mesto na Morave, 1983) (Prague), Charles Univ., 47-57.

[4] H. Gollek, The generalized sine-Gordon equation and its 1-soliton solutions, Ann. Global Anal. Geom., 3 (2), (1985), 233-264.

[5] K. Tenenblat and C.-L. Terng, Bäcklund's theorem for n-dimensional submanifolds of $R^{2 n-1}$, Ann. of Math., 111 (1980), 477-490.

[6] C.-L. Terng, A higher dimension generalization of the Sine-Gordon equation and its soliton theory, Ann. of Math., 111 (1980), 491-510.

Received August 18, 1992, revised November 30, 1992 and accepted for publication January 11, 1993.

\section{Lafayette College}

EASTON, PA 18042

E-mail address: buyskes@lafvax.lafayette.edu 




\section{PACIFIC JOURNAL OF MATHEMATICS}

Volume $166 \quad$ No. $2 \quad$ December 1994

Geometric aspects of Bäcklund transformations of Weingarten

submanifolds

STEVEN BUYSKE

Multipliers between invariant subspaces of the backward shift

225

ROBERT BRUCE CROFOOT

The Cauchy integral, analytic capacity and subsets of quasicircles

XIANG FANG

The number of lattice points within a contour and visible from the origin 295

Douglas Austin Hensley

On flatness of the Coxeter graph $E_{8}$

305

MASAKI IZUMI

Immersions up to joint-bordism

GUI SONG LI

Generalization of the Hilbert metric to the space of positive definite matrices

CARlangelo Liverani and Maciej WojtKowski

Periodicity, genera and Alexander polynomials of knots

SWATEE NAIK

On divisors of sums of integers. $\mathrm{V}$

ANDRÁS SÁRKÖZY and CAMERON LEIGH STEWART

Approximately inner automorphisms on inclusions of type $\mathrm{III}_{\lambda}$-factors

CARL WINSLøW

Correction to: "A convexity theorem for semisimple symmetric spaces"

KARL-HERMANN NEEB

Correction to: "Periodic points on nilmanifolds and solvmanifolds"

EDWARD KEPPELMANN

Correction to: "Partially measurable sets in measure spaces" 